\title{
Graph Rewriting and Strategies for Modeling Biochemical Networks
}

\author{
Oana Andrei ${ }^{1}$ and Hélène Kirchner ${ }^{2}$ \\ INRIA - LORIA \\ Campus scientifique BP 239 \\ F-54506 Vandoeuvre-lès-Nancy Cedex, France \\ Firstname.Lastnamedoria.fr
}

\begin{abstract}
In this paper, we present a rewriting framework for modeling molecular complexes, biochemical reaction rules, and generation of biochemical networks based on the representation of molecular complexes as a particular type of multigraphs with ports called molecular graphs. The advantage of this approach is to obtain for free a rewriting calculus which allows defining at the same level transformation rules and strategies for modeling rule selection and application, in order to prototype network generation.
\end{abstract}

\section{Introduction}

Many formal notations have already been used for modeling biological systems, and, in particular, protein-protein interactions concerned with the connectivity inside molecular complexes. These models can be classified as qualitative or quantitative, and the employed formal notations range from process calculi with different extensions or variations [10,14, 22], to term-rewriting [15] and graph rewriting [6].

In the biochemical model we consider, the behaviour of a protein is given by its functional domains that determine which other protein it can bind to or interact with. These domains are usually abstracted as sites that can be bound or free, visible or hidden. A protein is characterized by the collection of interaction sites on its surface. Proteins can bind to each other forming molecular complexes. Membranes can also form complexes, called tissues, due to the binding proteins on their surfaces. The structure of a complex is naturally described as a particular class of graphs, a mathematical structure that is easy to understand and use by computer scientists as well as by chemists and biologists.

We propose in this paper a calculus based on multigraph rewriting and rewrite strategies for modeling biochemical systems, in particular the structure and interactions of protein complexes. We use an extended version of multigraphs with ports [3] for describing the structure of complexes as molecular graphs and multigraph rewriting for modeling the interactions between them (Section 3). We encode the molecular graphs as terms, the reaction patterns as rewrite rules, and the transformations on molecular graphs as a rewriting relation (Section 4). We define a rewriting calculus for molecular graphs $\rho_{b i o}$-calculus, obtained from the rewrite calculus for labeled multigraphs with ports, the $\rho_{m g}$-calculus, introduced in [3], by adding state information on ports and 
imposing some conditions on edges. Strategic rewriting allows modeling the control mechanism in biochemical systems and the generation of biochemical networks. This is illustrated in Section 5 on a fragment of the epidermal growth factor receptor (EGFR) signaling cascade.

\section{Background}

In this section we briefly review some basic definitions of sorted term algebra, term rewriting [4] and strategic rewriting, graph theory, and labeled multigraphs with ports developed in [3].

Term Algebra. A many-sorted signature is a pair $(S, \Sigma)$, where $S$ is called the sort set and $\Sigma$ is an $S^{*} \times S$-sorted family $\left\{\Sigma_{w, s} \mid w \in S^{*}\right.$ and $\left.s \in S\right\}$. For $\mathcal{X}=\left\{\mathcal{X}_{s}\right\}$ an $S$-sorted family of disjoint sets of variables, $\mathcal{T}_{\Sigma}(\mathcal{X})$ is the smallest set of $\Sigma$-terms over $\mathcal{X}$ built with operators from $\Sigma$ and variables from $\mathcal{X}$. If $\mathcal{X}$ is empty, then we write $\mathcal{T}_{\Sigma}$ for $\mathcal{T}_{\Sigma}(\emptyset)$ and call it the set of ground $\Sigma$-terms. The set of all terms of sort $s$ is denoted by $\mathcal{T}_{\Sigma, s}(\mathcal{X})$. A ground substitution is a partial mapping from $\mathcal{X}$ to ground $\Sigma$-terms and it uniquely extends to a $\Sigma$-homomorphism from $\mathcal{T}_{\Sigma}(\mathcal{X})$ to $\mathcal{T}_{\Sigma}$. A finite ground substitution has the form $\sigma=\left\{x_{1} \mapsto t_{1}, \ldots, x_{n} \mapsto t_{n}\right\}$, with $x_{i} \in \mathcal{X}$ and $t \in \mathcal{T}_{\Sigma}$, for all $i=1$..n. Considering $S$ as a partially ordered set leads to quite similar definitions for order-sorted signature, terms and substitutions [19].

Term Rewriting. A set $\mathcal{R}$ of rewrite rules is a set of ordered pairs of terms of $\mathcal{T}_{\Sigma}(\mathcal{X})$, denoted $\ell \rightarrow r$, such that $l$ and $r$ belong to the same sort, $\ell \notin \mathcal{X}$ and $\operatorname{Var}(r) \subseteq \operatorname{Var}(\ell)$. In this paper, we only consider finite sets of rewrite rules. The rewriting relation induced by $\mathcal{R}$ is denoted by $\rightarrow_{\mathcal{R}}$ ( $\rightarrow$ if there is no ambiguity about $\mathcal{R}$ ), and defined by $s \rightarrow t$ iff there exists a substitution $\sigma$ and a position $p$ in $s$ such that $s=s[\sigma \ell]_{p}$ for some rule $\ell \rightarrow r$ of $\mathcal{R}$, and $t=s[\sigma r]_{p}$. This is written $s \rightarrow_{\mathcal{R}}^{p, \ell \rightarrow r, \sigma} t$ where either $\mathcal{R}, p, \ell \rightarrow r$, or $\sigma$ may be omitted. The reflexive transitive closure of the rewriting relation induced by $\mathcal{R}$ is denoted by $\stackrel{*}{\rightarrow}_{\mathcal{R}}$, and by $\stackrel{+}{\rightarrow}_{\mathcal{R}}$ its respective transitive closure. A rewriting derivation is a chain of terms $t_{1} \rightarrow t_{2} \rightarrow \ldots \rightarrow t_{n}$. The source of a derivation $t_{1} \rightarrow t_{2} \rightarrow \ldots$ is $t_{1}$, and when the derivation is finite, its last term is called the target.

A rewrite theory over $\mathcal{T}_{\Sigma}(\mathcal{X})$ is a triple $(\Sigma, E, \mathcal{R})$ where $E$ is a finite set of (sortpreserving) equalities and $\mathcal{R}$ a finite set of rewrite rules. The relation $\rightarrow_{\mathcal{R} / E}$ on $\mathcal{T}_{\Sigma}(\mathcal{X})$ is $=_{E} ; \rightarrow_{\mathcal{R}} ;={ }_{E}$ and it induces a relation $\rightarrow_{\mathcal{R} / E}$ on the quotient algebra $\mathcal{T}_{\Sigma}(\mathcal{X}) /={ }_{E}$ by $[t]_{E} \rightarrow_{\mathcal{R} / E}\left[t^{\prime}\right]_{E}$ iff $t \rightarrow_{\mathcal{R}, E} t^{\prime}$. The relation $\rightarrow_{\mathcal{R}, E}$ (called rewriting modulo $E$ ) on $\mathcal{T}_{\Sigma}(\mathcal{X})$ is defined by $s \rightarrow_{\mathcal{R}, E} t$ iff there exists a substitution $\sigma$ and a position $p$ in $s$ such that $\left.s\right|_{p}={ }_{E} \sigma l$ for some rule $l \rightarrow r$ of $\mathcal{R}$, and $t=s[\sigma r]_{p}$. In this paper, we will consider theories with $E$ defined by specific axioms, namely associativity (A), associativity and commutativity (AC), and unit element $(\mathrm{U})$ of some function symbols.

Strategic Rewriting. The notions of strategy and strategic rewriting have been introduced in order to control rewriting derivations. The notion of strategy can be defined in a general way: a rewrite strategy $\zeta$ for the rewrite system $\mathcal{R}$ is a subset of the set of all derivations of $\mathcal{R}$. A derivation of the strategy is called a strategic rewriting derivation. The application of a strategy $\zeta$ on a term $t$ is denoted $[\zeta](t)$ and defined as the set of all targets $t^{\prime}$ of the derivations of source $t$ in $\zeta$. When no derivation in $\zeta$ has $t$ as source, 


$$
\begin{aligned}
& {[\mathrm{id}](t)=\{t\}} \\
& {[\mathrm{fail}](t)=\emptyset} \\
& {[l \rightarrow r](t)=\left\{s \mid t \rightarrow^{l \rightarrow r} s\right\}} \\
& {\left[\text { onestep }\left(\zeta_{1}, \ldots, \zeta_{n}\right)\right](t)=\bigcup_{i}\left[\zeta_{i}\right](t)} \\
& {\left[\operatorname{seq}\left(\zeta_{1}, \zeta_{2}\right)\right](t)=\left[\zeta_{2}\right]\left(\left[\zeta_{1}\right](t)\right)} \\
& {\left[\operatorname{choice}\left(\zeta_{1}, \zeta_{2}\right)\right](t)=\left\{\begin{array}{l}
{\left[\zeta_{1}\right](t), \text { if }\left[\zeta_{1}\right](t) \neq \emptyset} \\
{\left[\zeta_{2}\right](t), \text { if }\left[\zeta_{1}\right](t)=\emptyset}
\end{array}\right.}
\end{aligned}
$$

Fig. 1. Strategy constructors

we say that the strategy application on $t$ fails. The result of the application of a failing strategy on a term $t$ is the empty set. We extend the application of a strategy to a set of terms as the union of the application of the strategy on each element of the set.

A strategy can be described by enumerating all its elements or more suitably by a strategy language. From elementary strategy expressions directly issued from a rewrite system $\mathcal{R}$, more elaborated strategy expressions are built like in ELAN [7], Stratego [25], TOM [5] or, more recently, Maude [16]. The semantics of such a language is naturally described in the rewriting calculus [12]. We describe below some constructs of the strategy language of interest in this paper and available in TOM.

Given a rewrite system $\mathcal{R}$ over $\mathcal{T}_{\Sigma}(\mathcal{X})$, a strategy expression is either a rewrite rule in $\mathcal{R}$ or an expression described below. A strategy operator $\zeta$ may take other strategies $\zeta_{1}, \ldots, \zeta_{n}$ as arguments, and the result is expressed functionally by $\zeta\left(\zeta_{1}, \ldots, \zeta_{n}\right)$. We present in Figure 1 some strategy constructors used in this paper. The strategy onestep computes all derivations issued from the application of a nonempty set of strategies. The strategy seq $\left(\zeta_{1}, \zeta_{2}\right)$ first applies $\zeta_{1}$ and then, if that succeeds, it applies $\zeta_{2}$; it fails if either $\zeta_{1}$ fails, or $\zeta_{2}$ fails. choice $\left(\zeta_{1}, \zeta_{2}\right)$ applies the strategy $\zeta_{1}$; if the application of $\zeta_{1}$ fails, it applies $\zeta_{2}$. Therefore choice $\left(\zeta_{1}, \zeta_{2}\right)$ fails if both $\zeta_{1}$ and $\zeta_{2}$ fail. Both strategy operators seq and choice extend naturally to be applicable to a list of strategies.

Along with the elementary rewrite rules, the strategy constructors represent the keycomponents necessary for defining more complex strategies. For instance, try and repeat allow iterating the application of a strategy and never fail.

$$
\begin{aligned}
& \operatorname{try}(\zeta)=\operatorname{choice}(\zeta, \text { id }) \\
& \operatorname{repeat}(\zeta)=\operatorname{try}(\operatorname{seq}(\zeta, \text { repeat }(\zeta)))
\end{aligned}
$$

Other high-level strategies implement term traversal and normalization on terms and are well-known in the term rewriting literature (see [5] for instance).

Labeled Graphs. A label alphabet $\mathcal{L}=\left(\mathcal{L}_{V}, \mathcal{L}_{E}\right)$ is a pair of sets of node labels and edge labels. A (finite) graph over $\mathcal{L}$ is a triple $G=(V, E, l)$ where $V$ is a (finite) set $\left\{v_{1}, \ldots, v_{n}\right\}$ of elements called nodes, $E$ is a family $\left(e_{1}, \ldots, e_{m}\right)$ of elements of the Cartesian product $V \times V$ called edges, and $l=\left(l_{V}, l_{E}\right)$ is the labeling function for nodes $\left(l_{V}: V \rightarrow \mathcal{L}_{V}\right)$ and edges $\left(l_{E}: E \rightarrow \mathcal{L}_{E}\right)$. If $G$ is a graph, we denote by $V_{G}$ its node set, by $E_{G}$ its edge set, and by $l_{G}$ its labeling function. An edge of the form $(v, v)$ is called a loop. For an edge $\left(v_{1}, v_{2}\right), v_{1}$ and $v_{2}$ are called end nodes (or end points) 


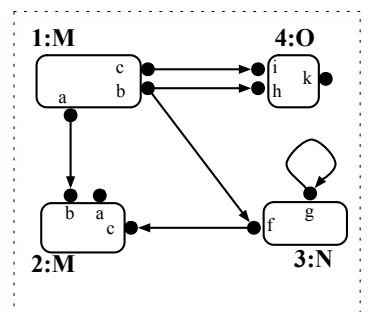

(a)

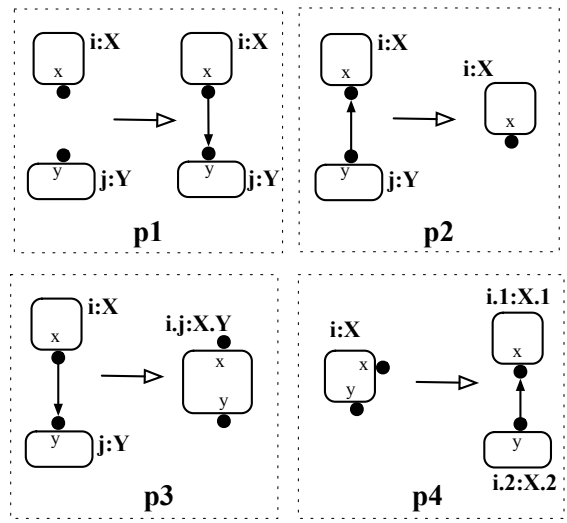

(b)

Fig. 2. A multigraph with ports (a) and some multigraph transformations (b)

with $v_{1}$ the source and $v_{2}$ the target; moreover we say that $v_{1}$ and $v_{2}$ are adjacent or neighboring nodes, with $v_{2}$ neighbor of $v_{1}$. An edge is incident to a node if the node is one of its end nodes. An edge is multiple if there is another edge with the same source and target; otherwise it is simple. A graph is simple if it has no multiple edges or loops, otherwise it is a multigraph. An adjacency list for a node is given by a list of pairs consisting of a neighbor and the corresponding edge label.

Labeled Multigraphs with Ports. A labeled multigraph with ports is obtained from a labeled multigraph by associating to each node a name and a set of ports such that each edge is determined by two ports, one from each end node. A node label consists of the node identifier, the name and the set of ports, while a simple edge label is the pair of source and target ports. Hereinafter we use multigraph instead of labeled multigraph with ports if there is no risk of confusion. We illustrate in Figure 2(a) such a multigraph.

Transforming Multigraphs with Ports. A transformation rule on multigraphs with ports $L \leadsto R$ consists of two multigraphs $L$ and $R$ called the left- and right-hand side respectively, and a correspondence between nodes of $L$ and nodes of $R, \xi: V_{L} \rightarrow \mathcal{P}\left(V_{R}\right)$, which we call node-substitution. This correspondence is provided by the unique node identifiers. The application of a multigraph transformation rule $L \leadsto R$ to a multigraph $G$ produces a new multigraph $G^{\prime}$ by the following steps: first, find and remove a subgraph of $G$ isomorphic with $L$ via a matching morphism $m$, resulting in a context graph $G^{-}$; then add and reconnect $m(R)$ to $G^{-}$with the help of $m(\xi)$.

We illustrate in Figure 2 (b) some simple multigraph transformations: p1 creates an edge between two ports with $\xi(i)=\{i\}$ and $\xi(j)=\{j\}$, p2 removes the source node of an edge with $\xi(i)=\{i\}$ and $\xi(j)=\emptyset, \mathbf{p 3}$ merges two adjacent nodes with $\xi(i)=\xi(j)=\{i \cdot j\}$, and $\mathbf{p} 4$ splits a node containing (at least) two ports in two adjacent nodes by distributing a port to each node with $\xi(i)=\{i .1, i .2\}$.

The Rewriting Calculus for Multigraphs with Ports. The rewriting calculus for labeled multigraphs with ports (or $\rho_{m g}$-calculus), fully detailed in [3], is a variation of the 
$\rho$-calculus [12] with particular features related to multigraph transformation and it provides a clear operational semantics to multigraph transformation. We benefit from the capabilities of $\rho$-calculus of encoding rewrite strategies and conditional rewrite rules. Moreover the $\rho$-calculus integrates $\lambda$-calculus and rewriting in a powerful higher-order pattern calculus.

\section{The Biochemical Model}

A molecular complex consists of molecules connected through bonds between sites. A molecule is characterized by a pair of name and set of sites. In order to make a distinction between molecules having the same name, we assign to each molecule a unique identifier consisting of a sequence of integers. Each site is characterized by $\mathrm{a}$ name and a state: $\mathrm{b}$ for bound, $\mathrm{v}$ and $\mathrm{h}$ for free sites that are visible and hidden respectively.

We represent a molecular complex as a connected labeled multigraph with ports where molecules are nodes, sites are ports with states, and bonds are simple edges, together with the restriction that a port can be the end node of at most one edge. We call this kind of multigraphs, not necessarily connected, molecular graphs. In Figure 3 we define their abstract syntax, with "," the juxtaposition operator and $\epsilon$ the empty set. An unbound molecule has also a molecular graph representation as a single node with an empty list of bonds.

Using the same graphical representation as for multigraphs, inspired by [6, 14], we represent a molecule as an empty box having the identifier placed at the exterior and the sites as small points on the surface of the box. We represent the state of a site as follows: a filled circle for bound sites, an empty one for visible sites, and a slashed circle for hidden sites.

A biochemical system is represented as a discrete system consisting of interacting components which give rise to structural and behavioural transformation of the components and of the system as a whole. Such a system is dynamic, has an emergent behaviour, is highly concurrent and non-deterministic.

The interactions are abstracted as reaction patterns: each of the reaction patterns corresponds to a class of reactions characterized by common features. There are two types of protein interactions: (i) state changing by attaching or removing small phosphate groups at specific sites, and (ii) complexation by binding sites or decomplexation

\begin{aligned} & \hline Id $i::=$ Int $^{+} \\ &$Name $m, s:=$ String \\ & State $t::=\mathrm{b}|\mathrm{v}| \mathrm{h} \\ &$ Sites $S::=s \frown t|S, S| \epsilon \\ &$ Molecules $M::=\langle i: m \| S\rangle|M, M| \epsilon \\ &$ Bonds $B::=(i \frown s, i \frown s)|B, B| \epsilon \\ &$ Molecular graph $G::=(M, B) \\ &$\hline\end{aligned}

Fig. 3. The syntax of molecular graphs 
by breaking a bond between two sites. An interaction may reveal new sites or hide some of the existing ones.

Given an initial set of biochemical species (as molecular graphs) and reactions described by a set of patterns (as molecular graph transformation rules), we obtain a network by iteratively applying the reactions on the existing biochemical species until a termination condition is satisfied or until the exhaustive generation of all possible species is achieved.

Example 1. We illustrate a fragment of the EGFR signaling cascade already formalized in papers like $[14,22]$. The protagonists of this model, depicted below, are: the signal protein EGF situated outside the cell acting as a ligand, the transmembrane protein EGFR with two extracellular sites and two intracellular sites as a receptor, and the adapter protein $\mathrm{SHC}$ inside the cell.

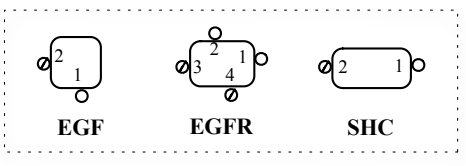

The signaling information is propagated from outside the cell to its interior following the reaction patterns depicted in Figure 4. We note that, for the molecules participating in the reaction patterns, only the relevant sites are made precise, the rest of them remain unchanged. The ligand EGF does not enter the cell, instead it transmits the information as a signal across the cell membrane. The function of information transfer across the membrane is performed by the receptor EGFR. One extracellular binding site of the receptor recognizes the signal protein in a dimeric form EGF.EGF produced by (r1) leading to the creation of a bond between the EGF dimer and the receptor, and

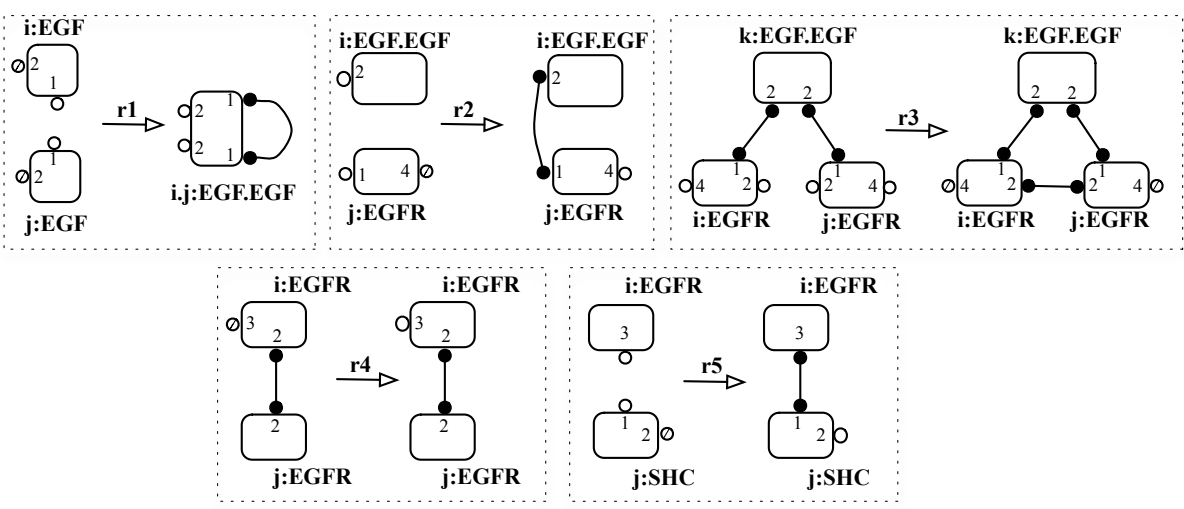

Fig. 4. The reaction patterns in the EGFR signaling cascade fragment 
to the activation by phosphorylation of a second extracellular site of the receptor (r2). Two receptors activated by the same EGF dimer bind by (r3) creating an active dimer - a receptor tyrosine kinase (RTK). The RTK attaches phosphate groups to certain tyrosines, first on itself $(\mathbf{r 4})$, and then on other proteins by activating them, as it is the case for the adapter $S H C$ (r5). Some of these latter proteins are also tyrosine kinases and, consequently, a cascade of phosphorylations occurs. We illustrate in Figure 5 an initial molecular graph $\mathbf{G}$ we consider for this example, and two molecular graphs $\mathbf{G}$ ' and $\mathbf{G}$ " resulting from $\mathbf{G}$ by applying some of the reactions above.

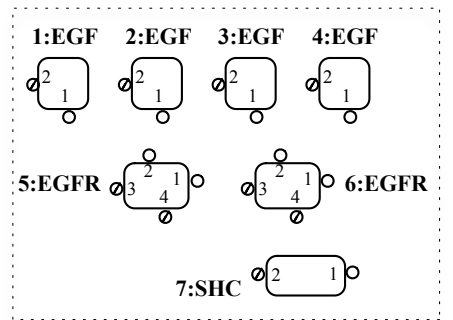

$\mathbf{G}$

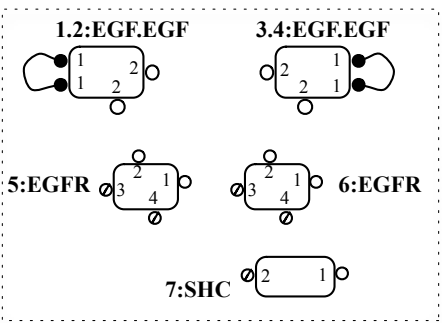

$\mathbf{G}^{\prime}$

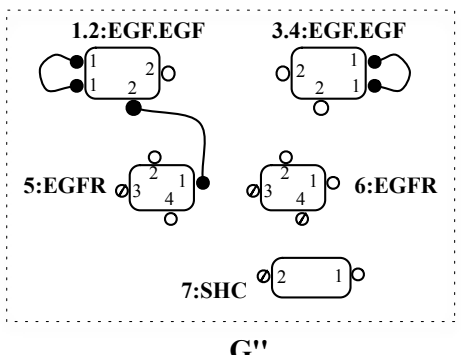

$\mathbf{G}^{\prime \prime}$

Fig. 5. G is the initial configuration; G' results from applying twice $\mathbf{r} \mathbf{1}$ on $\mathbf{G}$; G' results from an application of $\mathbf{r} \mathbf{2}$ on $\mathbf{G}$ '

After the initiation of the signaling process, the signaling cascade must be terminated at a certain point since cells must be able to stop responding to a signal. If not, the signaling process may lead to uncontrolled cell growth as, for instance, in cancer. This process is ended by quickly engulfing and destroying the ligand-receptor complex by receptor-mediated endocytosis.

\section{Term Rewriting Semantics for Molecular Graphs Transformations}

We present in this section a signature for terms encoding molecular graphs, an encoding of reaction patterns as rewriting rules, and a rewriting relation encoding the application of reactions patterns on molecular graphs. 


\subsection{Encoding the Molecular Graphs}

We encode molecular graphs as terms using an order-sorted signature $\Sigma=(\mathcal{S},<, \mathcal{F})$ similarly to the term rewriting encoding of multigraphs with ports [3] up to sort renaming and extension of site information with a state. We give here only the operator set:

$$
\begin{aligned}
& \epsilon_{\mathrm{X}} \quad: \longrightarrow \mathrm{X} \text { Set } \\
& { }_{-}, \quad: \mathrm{XSet} \times \mathrm{XSet} \longrightarrow \mathrm{XSet}\left[A C U\left(\epsilon_{\mathrm{X}}\right)\right] \\
& \therefore \quad: \text { SiteName } \times\{\mathrm{b}, \mathrm{v}, \mathrm{h}\} \rightarrow \text { Site } \\
& \left\langle\iota_{-} \text {_ }_{-}{ }_{-}\right\rangle: I d \times \text { MoleculeName } \times \text { SiteSet } \longrightarrow \text { Molecule } \\
& \left({ }_{-},-\right) \quad: \text { SiteName } \times \text { SiteName } \longrightarrow \text { Bond } \\
& \text { - } \_: \text {Id } \times \text { BondSet } \longrightarrow \text { Neighbor } \\
& { }_{-} \_\quad: I d \times \text { NeighborSet } \longrightarrow \text { AdjacencyEq } \\
& \text {-(-D) : MoleculeSet } \times \text { AdjacencyEqSet } \longrightarrow \text { MGraph }
\end{aligned}
$$

where $\mathrm{X}$ takes sort values from the set $\{$ Molecule, Bond, Neighbor, AdjacencyEq\}.

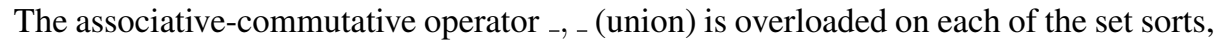
and $\epsilon_{\mathrm{X}}$ denotes the the empty set. We use $\epsilon$ instead of $\epsilon_{\mathrm{X}}$ whenever the sort X can be easily deduced from the context. The subsorting relation $\mathrm{X}<\mathrm{XSet}$ states that each term of sort $\mathrm{X}$ can be seen as a set with a single element. The axiomatizing involves theories with associativity, commutativity and unit element axioms (ACU) for the constructors _, _ of each set-like sort with $\epsilon$ the unit.

Since the molecular graphs represent a particular case of multigraphs with ports and we encoded them in the oriented version, we impose an orientation of bonds by choosing an arbitrary order $\succ$ on molecule names in the model and by considering that:

- a bond between two molecules of different name corresponds to a bond with the source labeled by the greater molecule name and the target labeled by the smaller molecule name, and

- a bond between two molecules of the same name is not oriented (or we can represent it equivalently as a bidirectional edge).

Sometimes biochemical reactions may occur without knowing all intermediate states or molecule names. This motivates us in using "unknown" names for molecules and sites as in [24] for beta-binders. We then choose to represent a bond between two molecules where at least one of them has an unknown name by an unoriented bond (or, equivalently, a bidirectional bond), and call this assumption supra-estimation. When a new molecule name is produced by a reaction, the total order on names is extended for including it. One simple way to handle this extension is to consider any new name to be smaller than all existing names.

Let $\mathcal{X}$ be an $(\mathcal{S},<)$-sorted family of variables.

Definition 2. We encode a molecular graph $G=(M, B)$ as an algebraic term $t_{1}\left(t_{2}\right)$ of sort MGraph where $t_{1} \in \mathcal{T}_{\Sigma, \text { MoleculeSet }}(\mathcal{X})$ encodes the set of all molecules in $G$, and $t_{2} \in \mathcal{T}_{\Sigma}$,AdjacencyEqSet $(\mathcal{X})$ encodes the set of adjacency equations providing the neighbors for each molecule in $M$ (if any) together with the labels of the incident bonds.

Algebraic terms encoding molecular graphs must satisfy some structural properties in order to be considered well-formed: (i) each molecule identifier occurs at most once: 
in the molecule set, in the adjacency equation set as left-hand side of an adjacency equation, in the neighbor set of a molecule identifier; (ii) each molecule identifier or site occurring in the adjacency equation set must also occur in the molecule set; (iii) each site has at most one incident bond and its state is correct w.r.t. the incidence information provided in the adjacency equation set. Additionally, these terms must satisfy some canonical form requirements: (i) right-hand sides of adjacency equations are non-empty sets of neighbors; (ii) only non-empty set of bonds occur in neighbor terms.

Example 3. The molecular graph occurring in the left-hand side of the reaction pattern r3 illustrated in Figure 4 is encoded as the following term, imposing an edge orientation given by the following total order on molecule names EGFR $\succ E G F \succ S H C \succ$ EGF.EGF:

$$
\begin{aligned}
& \left(\left\langle i: E G F R \| 1^{\wedge} \mathrm{b}, 2^{\wedge} \mathrm{v}, 4^{\wedge} \mathrm{v}\right\rangle,\left\langle j: E G F R \| 1^{\wedge} \mathrm{b}, 2^{\wedge} \mathrm{v}, 4^{\wedge} \mathrm{v}\right\rangle,\right. \\
& \left.\left\langle k: E G F . E G F \| 2^{\wedge} \mathrm{b}, 2^{\wedge} \mathrm{b}\right\rangle\right)\left(i \bumpeq k^{\frown}(1,2), j \bumpeq k \frown(1,2)\right)
\end{aligned}
$$

\subsection{Encoding the Reaction Patterns}

For all rewrite rules over $\mathcal{T}_{\Sigma, M G r a p h}(\mathcal{X})$ we impose molecule identifiers occurring in the left-hand side to be variables. We say that a rewrite rule $t_{1} \rightarrow t_{2}$ over $\mathcal{T}_{\Sigma, M G r a p h}(\mathcal{X})$ is well-formed (in canonical form) if both $t_{1}$ and $t_{2}$ are well-formed (in canonical form respectively). We call bio-rewrite rule a well-formed rewrite rule in canonical form.

Definition 4. We encode a molecular graph rewrite rule $G_{1} \leadsto G_{2}$ as a term rewrite rule $_{1} \rightarrow t_{2}$ where $t_{i}$ encodes $G_{i}$, for $i=1,2$.

The encoding of a molecular graph rewrite rule is a bio-rewrite rule since by definition the term encoding of a molecular graph is well-formed and in canonical form.

The node-substitution corresponding to a bio-rewrite rule, like for the encoding of transformations on multigraphs with ports, can be extracted automatically by means of a procedure GetMap analyzing the identifier occurrences of both sides of a rule. We make explicit the deletion of a molecule (site) in the node-substitution by mapping it to a new molecule (site) $\bullet$ called black hole.

Example 5. The molecular graph transformation $\mathbf{r} 1$ illustrated in Figure 4 has the following encoding as a rewrite rule:

$$
\begin{aligned}
& \left(\left\langle i: E G F \| 1^{\wedge} \mathrm{v}, 2^{\wedge} \mathrm{h}\right\rangle,\left\langle j: E G F \| 1^{\wedge} \mathrm{v}, 2^{\wedge} \mathrm{h}\right\rangle\right)(\epsilon) \rightarrow \\
& \left\langle i . j: E G F . E G F \| 1^{\wedge} \mathrm{b}, 1^{\wedge} \mathrm{b}, 2^{\wedge} \mathrm{v}, 2^{\wedge} \mathrm{v}\right\rangle(i . j \bumpeq i . j \frown(1,1))
\end{aligned}
$$

with the corresponding node-substitution:

$$
\begin{aligned}
& \left\langle i: E G F \| 1^{\wedge} \mathrm{v}, 2^{\wedge} \mathrm{h}\right\rangle \mapsto\left\langle i \cdot j: E G F . E G F \| 1^{\wedge} \mathrm{b}, 1^{\wedge} \mathrm{b}, 2^{\wedge} \mathrm{v}, 2^{\wedge} \mathrm{v}\right\rangle, \\
& \left\langle j: E G F \| 1^{\wedge} \mathrm{v}, 2^{\wedge} \mathrm{h}\right\rangle \mapsto\left\langle i \cdot j: E G F . E G F \| 1^{\wedge} \mathrm{b}, 1^{\wedge} \mathrm{b}, 2^{\wedge} \mathrm{v}, 2^{\wedge} \mathrm{v}\right\rangle
\end{aligned}
$$




\subsection{Encoding the Reaction Pattern Application}

The rewriting relation is the encoding of the multigraph transformation as presented in Section 2 with some particularities given by the instantiation of multigraphs with ports as molecular graphs.

The main ideas behind a rewriting step $t \stackrel{\bar{r}}{\rightarrow} t^{\prime}$ with $r: t_{1} \rightarrow t_{2}$ are the following:

$-\bar{r}: \overline{t_{1} \rightarrow t_{2}}$ is the extended rule obtained from $r$ by appending extension variables to each set-sorted subterm in the left-hand side and, accordingly, in the right-hand side.

- GetMap computes for a rewrite rule on MGraph-sorted terms the associated nodesubstitution using an analysis on the identifier occurrences.

- We use an ACU matching algorithm together with a matching rule which allows us to solve match equations where the left hand-side is a NeighborSet- or BondSetsorted term $t_{1}, \ldots, t_{k}$ (with $k \geq 2$ ) not containing extension variables, and the right-hand side is a singleton, $t$.

Such cases arise due to supra-estimated bonds. The problem is solved by matching only one term $t_{i}$ from the left-hand side against the term $t$ from the right-hand side, discarding the other terms $t_{j}$, with $j \neq i$, and matching the extension variables against the set unit $\epsilon$.

- We apply the substitution $\sigma$ as usual. However, the result of the application of a substitution $\{x \mapsto t\}$ with $x, t:$ MoleculeId on adjacency equations requires a special treatment. For example, let us assume by supra-estimation that the nodes represented by $x$ and $y$ are connected by a bidirectional bond. If, after the application of a substitution $\sigma$, the name of $\sigma(y)$ is greater than the name of $\sigma(x)$, then the bond becomes unidirectional with $\sigma(x)$ the target and $\sigma(y)$ the source of the bond.

- We apply the instantiated node-substitution $\xi^{\sigma}$ on $\sigma\left(\overline{t_{2}}\right)$ by sequentially applying each of its elementary node-substitution on the term. The application of a nodesubstitution on an adjacency equation (or a neighbor) transforms it in $n$ adjacency equations (resp. neighbors), one for each corresponding node in the right-hand side of the mapping, and propagates the mapping application on the set of neighbors. After applying a node-substitution, the $M G r a p h$-terms may be neither well-formed, nor in canonical form.

- $\mathcal{S}$ is the rewrite system defining transformations on terms into well-formed MGraphsorted terms in canonical form. Such rules (i) delete the adjacency equations for black holes and the black hole neighbors taking care of updating the states of incident sites, (ii) delete the extra-bonds (bonds whose endpoints do not appear among the sites of the connected nodes), (iii) merge elements having the same identifier, and (iv) delete neighbors with empty bond sets and adjacency equations with an empty neighbor sets.

Definition 6. A term $t$ of sort $M G r a p h$ rewrites to a term $t^{\prime}$ using a bio-rewrite rule $r: t_{1} \rightarrow t_{2}$ where $t_{1}, t_{2} \in \mathcal{T}_{\Sigma, M G r a p h}(\mathcal{X})$ with $\bar{r}: \overline{t_{1} \rightarrow t_{2}}$ and $\xi=\operatorname{GetMap}(\bar{r})$, if there exists a substitution $\sigma$ solution of the ACU-matching problem $\overline{t_{1}} \ll t$ such that $t^{\prime}=\xi^{\sigma}\left(\sigma\left(\overline{t_{2}}\right)\right) \downarrow_{\mathcal{S}}$. We call this relation bio-rewriting, and we say that $t$ bio-rewrites to $t^{\prime}$ by $r$ and denote it by $t \stackrel{\bar{r}}{\rightarrow} t^{\prime}$. 
We do not illustrate here an example of a bio-rewriting step since it would be too technical (see [3] for such an example). However, it is important to emphasize that, according to the correspondence theorem in [3], the rewriting relation defined here on $M G r a p h$-terms encodes the transformation relation on molecular graphs.

\section{$4.4 \rho_{b i o}$-Calculus}

Following closely the same steps for encoding the more general multigraphs with ports, we obtain for free a high-level calculus for molecular complexes which we call $\rho_{b i o^{-}}$ calculus, similar to the $\rho_{m g}$-calculus. By extending the $\lambda$-calculus and term rewriting, the $\rho_{b i o}$-calculus provides a way to combine rewriting and high-order functions. In addition, it fits in the direction given by Fontana and Buss [18] of using the $\lambda$-calculus as a formalism for studying biochemical systems.

\section{Biochemical Network Generation}

A biochemical system is not completely described by its components and the way they interact by means of reactions, but also by the behaviour of the system as a whole. Modeling the generation of a biochemical network amounts to defining how a set of reaction patterns is applied on a collection (set or multiset) of molecules. Strategic rewriting provides a formal model for expressing the control on the reaction rule application.

One rough way of describing the behaviour of the EGFR signaling cascade is to try to apply repeatedly all reaction patterns on the initial collection of molecules (see $\zeta_{1}$ in Figure 6). We can easily prove that this strategy terminates since the number of free binding sites decreases or remains constant with every rule application.

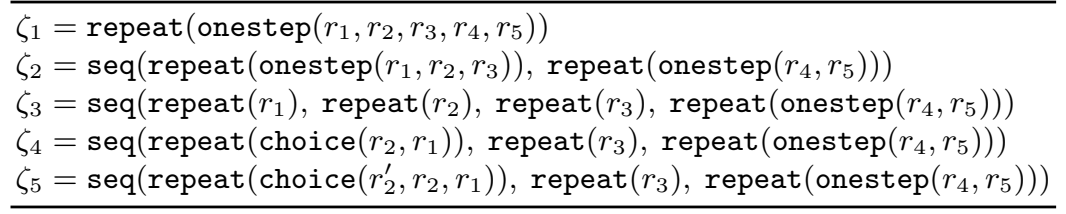

Fig. 6. Different strategies for the modeling the network generation

The execution can be separated in two stages: the first one, $\left\{r_{1}, r_{2}, r_{3}\right\}$, is concerned with the extracellular interactions between the signals and the receptors, while the second one, $\left\{r_{4}, r_{5}\right\}$, with the RTK cascade. This is specified by the strategy $\zeta_{2}$ as a repeated sequential composition of two strategies, one for each stage. The strategy $\zeta_{3}$ specifies that all EGF dimers are created in a first step, and only after that they bind to receptors, while the strategy $\zeta_{4}$ specifies that an EGF dimeric form binds a receptor as soon as it is created by giving $r_{2}$ priority over $r_{1}$ using the choice strategy.

In order to disallow the occurrence of molecular graphs like the one in Figure 7(a) where each of the EGF dimeric forms binds one of the receptor and the binding of 


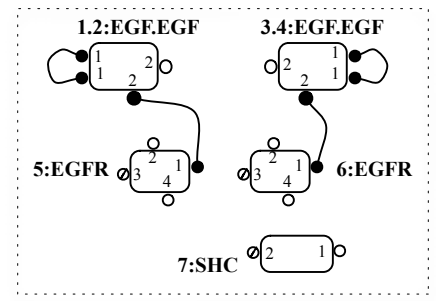

(a)

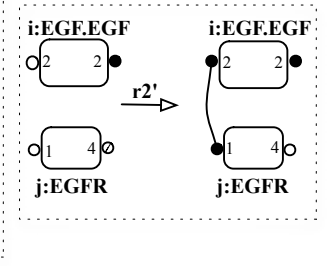

(b)

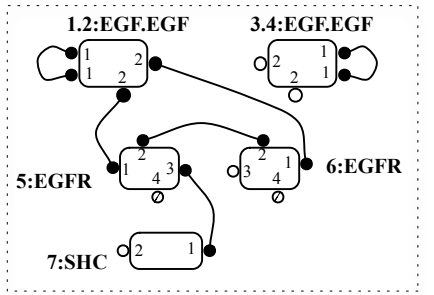

Fig. 7. (a) A possible graph obtained from applying $\mathbf{r} 2$ on G" from Figure 5. (b) An additional rule which urges the binding of a dimeric form of EGF to a second receptor. (c) A molecular graph resulting from applying the strategy $\zeta_{5}$ on the initial molecular graph from Figure 5.

receptors is no longer possible, we introduce a new reaction rule r2' depicted in Figure 7(b). Providing $\mathbf{r 2}$ ' with a higher priority than $\mathbf{r} \mathbf{2}$ corresponds to a dimerization of the receptors as soon as possible. The priority relation is modeled by the strategy choice $\left(r_{2}^{\prime}, r_{2}\right)$ which tries to apply $r_{2}^{\prime}$ first, and only if this does not succeed, it applies the rule $r_{2}$. Hence the EGF dimer binds two receptors as soon as it is created by means of the strategy $\zeta_{5}$. This last strategy applied on the initial molecular graph $\mathbf{G}$ illustrated in Figure 5 produces the molecular graph from Figure 7(c) modulo a relabeling of the dimeric forms of EGF. Usually we obtain an exhaustive generation of the network by repeating the application of the control strategies, for instance repeat $\left(\zeta_{i}\right)$, for $i \in\{2, \ldots, 5\}$.

The use of strategies at this stage provides our calculus with a declarative formalism for modeling a flexible control of rule applications. Different control strategies may be easily prototyped and experimented by selecting specific rules, grouping rules and applying each group with different strategies, and then linking them up. The advantages of using strategic rewriting for the network generation have already been experimented in $[8,2]$ for a chemical application.

\section{Conclusion}

An inspiring starting point for our work was the graphical formalism presented in [6] for modeling biochemical networks where the protein complexes are represented by typed attributed graphs, and classes of reactions are modeled by graph transformation rules. This model considers also quantitative information on reactions. Our approach, developed on a rule-based modeling framework and extensively using expressive graphical representations (like the one of [6]), focuses on providing in addition a strategic rewriting dimension. This new component allows a flexible modeling for the generation of biochemical networks.

The $\rho_{b i o}$-calculus introduced in this paper for modeling the interactions between molecular complexes is an extension of the $\rho_{m g}$-calculus [3]. The connectivity information in molecular complexes is essential in the definition of some reaction patterns since it can directly affect their reactivity; this kind of information is easily modeled by 
graph transformation rules. It is also possible to express negative constraints in the term encoding of the reaction patterns by means of recently developed anti-patterns [21], a concept already implemented in TOM. This allows us to specify for instance the absence of certain molecules or forbidden bonds, and hence to model halting conditions as forbidden occurrences of some particular molecular graphs.

Using graph rewriting and rule-based formalisms for modeling biological systems has already been done by several authors. A review of such rule-based formalisms can be found in [20] with emphasis on the capability of representing the topology of complexes. $\kappa$-calculus [14] is a language of formal proteins which models complexes as graphs-with-sites and their interactions as a particular graph-rewriting operation. It is derived from process calculus, and bonds are represented in complexes by shared names. A step forward, bio $\kappa$-calculus [22] combines the $\kappa$-calculus with the brane calculi [9] providing a more expressive formalism by modeling the effects of protein interactions on the interaction capabilities of membranes. In this paper, we initiate the development of a similar approach based on a different calculus.

Pathway Logic [15] is a rewriting system formalism, where proteins and cells are modeled by algebraic terms, and reactions by rewrite rules. It is designed to work on two levels of abstraction, one concerning the protein states, and the other concerning the protein-protein interactions handled by means of graph rewriting. The Maude [13] system is used for implementing Pathway Logic, providing executability of the specifications and analytic tools. As in Pathway Logic, we use algebraic terms and rewrite rules for modeling molecules and reactions respectively, with the differences that we represent graphs using adjacency lists, and the control of rewrite rule applications is expressed inside the calculus.

Based on our previous experience on implementing molecular chemistry [8, 2], we are currently implementing this calculus using TOM, a tool providing matching, normalization, and strategic rewriting in high-level programming languages like Java. An aspect we cannot neglect in modeling protein-protein interactions is the combinatorial complexity [20]. In order to obtain an efficient implementation, we make extensive use of the maximal term sharing provided by TOM.

Several further extensions of this work are possible:

We have conceived a qualitative model; by imposing kinetic information on reactions and on the initial set of biochemical species we should obtain biochemical networks allowing us to derive quantitative models as ODE-based models, stoichiometric models, time depending. An inspiring rule-based formalism for a qualitative modeling of biochemical processes is found in BIOCHAM [11].

We focus in this paper on interactions between proteins at the level of functional domains. However, the model we propose can be easily tuned to represent other types of biomolecular interactions, such as protein-DNA or protein-lipid interactions [17], or even membrane interactions $[9,10]$.

Strategic rewriting is a suitable formalism for modeling the highly concurrent and non-deterministic behaviour of complex systems in general; in particular, the use of strategic rewriting makes possible to describe biochemical processes by expressions in a suitable strategy language. A first link between strategies and computation models 
inspired by biology is presented in [1] where control mechanisms in membrane systems are expressed by means of rewrite strategies.

Strategic rewriting opens many possibilities for modeling different aspects of biological systems at different abstraction levels. In particular, we plan to explore the capabilities of this formalism to model and reason about the adaptability and flexibility of cell behaviour. We do not limit our aim to modeling some well-known biological systems, but to help understand their behaviour and deduce new organization and behavioural principles. In the same vein as Păun in [23], we consider that reasoning at the level of strategies of computing (rewrite strategies), rather than at the tactic level (rewrite rules), is an incentive direction of formally studying biological systems.

\section{References}

1. O. Andrei, G. Ciobanu, and D. Lucanu. Expressing Control Mechanisms in P systems by Rewriting Strategies. In H. Hoogeboom, G. Paun, G. Rozenberg, and A. Salomaa, editors, Membrane Computing, International Workshop, WMC7, Leiden, The Netherlands, 2006, Selected and Invited Papers, volume 4361 of Lecture Notes in Computer Science, pages 154169. Springer, 2006.

2. O. Andrei, L. Ibanescu, and H. Kirchner. Non-intrusive Formal Methods and Strategic Rewriting for a Chemical Application. In K. Futatsugi, J.-P. Jouannaud, and J. Meseguer, editors, Algebra, Meaning, and Computation, Essays Dedicated to Joseph A. Goguen on the Occasion of His 65th Birthday, volume 4060 of Lecture Notes in Computer Science, pages 194-215. Springer, 2006.

3. O. Andrei and H. Kirchner. A Rewriting Calculus for Multigraphs with Ports. In Proceedings of RULE'07, 2007.

4. F. Baader and T. Nipkow. Term Rewriting and All That. Cambridge University Press, 1998.

5. E. Balland, P. Brauner, R. Kopetz, P.-E. Moreau, and A. Reilles. Tom: Piggybacking Rewriting on Java. In F. Baader, editor, RTA'07, volume 4533 of Lecture Notes in Computer Science, pages 36-47. Springer, 2007.

6. M. L. Blinov, J. Yang, J. R. Faeder, and W. S. Hlavacek. Graph Theory for Rule-Based Modeling of Biochemical Networks. In C. Priami, A. Ingólfsdóttir, B. Mishra, and H. R. Nielson, editors, Transactions on Computational Systems Biology VII, volume 4230 of Lecture Notes in Computer Science, pages 89-106. Springer, 2006.

7. P. Borovanský, C. Kirchner, H. Kirchner, P.-E. Moreau, and C. Ringeissen. An overview of ELAN. Electronic Notes in Theoretical Computer Science, 15, 1998.

8. O. Bournez, G.-M. Côme, V. Conraud, H. Kirchner, and L. Ibanescu. A Rule-Based Approach for Automated Generation of Kinetic Chemical Mechanisms. In R. Nieuwenhuis, editor, Rewriting Techniques and Applications (RTA 2003), volume 2706 of Lecture Notes in Computer Science, pages 30-45. Springer, 2003.

9. L. Cardelli. Brane Calculi. In V. Danos and V. Schächter, editors, Computational Methods in Systems Biology, International Conference CMSB 2004, Paris, France, May 26-28, 2004, Revised Selected Papers, volume 3082 of Lecture Notes in Computer Science, pages 257278. Springer, 2005.

10. L. Cardelli and S. Pradalier. Where Membranes Meet Complexes. In Proceedings of BioCONCUR 2005: A workshop on concurrent models in molecular biology, 2005.

11. N. Chabrier-Rivier, F. Fages, and S. Soliman. The Biochemical Abstract Machine BIOCHAM. In V. Danos and V. Schächter, editors, Computational Methods in Systems 
Biology, International Conference CMSB 2004, Paris, France, May 26-28, 2004, Revised Selected Papers, volume 3082 of Lecture Notes in Computer Science, pages 172-191. Springer, 2005.

12. H. Cirstea and C. Kirchner. The rewriting calculus - Part I and II. Logic Journal of the IGPL, 9(3):427—498, 2001.

13. M. Clavel, F. Durán, S. Eker, P. Lincoln, N. Martí-Oliet, J. Meseguer, and J. F. Quesada. Maude: specification and programming in rewriting logic. Theoretical Computer Science, 285(2):187-243, 2002.

14. V. Danos and C. Laneve. Formal molecular biology. Theoretical Computer Science, 325(1):69-110, 2004.

15. S. Eker, M. Knapp, K. Laderoute, P. Lincoln, and C. L. Talcott. Pathway Logic: Executable Models of Biological Networks. Electronic Notes in Theoretical Computer Science, 71, 2002.

16. S. Eker, N. Martí-Oliet, J. Meseguer, and A. Verdejo. Deduction, Strategies, and Rewriting. Electronic Notes in Theoretical Computer Science, 174(11):3-25, 2007.

17. J. R. Faeder, M. L. Blinov, and W. S. Hlavacek. Graphical rule-based representation of signaltransduction networks. In H. Haddad, L. M. Liebrock, A. Omicini, and R. L. Wainwright, editors, Proceedings of the 2005 ACM Symposium on Applied Computing (SAC), Santa Fe, New Mexico, USA, pages 133-140. ACM, 2005.

18. W. Fontana and L. W. Buss. The barrier of objects: From dynamical systems to bounded organizations. In J. Casti and A. Karlqvist, editors, Boundaries and Barriers., pages 56-116. Addison-Wesley, 1996.

19. J. A. Goguen and J. Meseguer. Order-Sorted Algebra I: Equational Deduction for Multiple Inheritance, Overloading, Exceptions and Partial Operations. Theoretical Computer Science, 105(2):217-273, 1992.

20. W. S. Hlavacek, J. R. Faeder, M. L. Blinov, R. G. Posner, M. Hucka, and W. Fontana. Rules for Modeling Signal-Transduction Systems. Science STKE 334, 2006.

21. C. Kirchner, R. Kopetz, and P.-E. Moreau. Anti-Pattern Matching. In Proceedings of the 16th European Symposium on Programming - ESOP'07, volume 4421 of Lecture Notes in Computer Science, pages 110-124. Springer, 2007.

22. C. Laneve and F. Tarissan. A simple calculus for proteins and cells. Electronic Notes in Theoretical Computer Science, 171(2):139-154, 2007.

23. G. Paun. Twenty Six Research Topics About Spiking Neural P Systems. Available at the P Systems Web Page: http: / / psystems. disco.unimib.it., 2006.

24. C. Priami and P. Quaglia. Beta Binders for Biological Interactions. In V. Danos and V. Schächter, editors, Computational Methods in Systems Biology, International Conference CMSB 2004, Paris, France, May 26-28, 2004, Revised Selected Papers, volume 3028 of Lecture Notes in Computer Science, pages 20-33. Springer, 2004.

25. E. Visser, Z.-E.-A. Benaissa, and A. P. Tolmach. Building Program Optimizers with Rewriting Strategies. In ICFP, pages 13-26, 1998. 